\title{
Cumul des impensables : le second cancer
}

\section{Overcome by the Unthinkable: a Second Cancer}

\section{M.-F. Bacqué · A. Polomeni · M. Derzelle \\ (C) Lavoisier SAS 2018 \\ Retour des étiologies primitives : mauvais sort, superstition}

«Pas de chance! » semble l'exclamation lancée spontanément par les patients lorsqu'ils découvrent le diagnostic d'un deuxième cancer après le traitement du premier. Alice Polomeni, à l'origine de ce dossier " second cancer » rapporte comment sa survenue fait l'objet d'incrédulité, d'un bouleversement parfois plus intense que lors de l'annonce du premier. Pour les psychologues et les médecins en oncologie, le redoublement du cancer produit un effet crescendo dans ce que peut supporter une personne, un patient, une équipe soignante. Pourtant, ces seconds cancers ne sont pas si rares, en raison de la longévité de la population et de la survie des patients plus ou moins jeunes (personnes de plus de 50 ans principalement, mais aussi bébés, enfants atteints de cancer parfois), soignés pour ces pathologies.

\section{Qu'est-ce qu'un second cancer primitif ?}

D'après le rapport de l'INCa de 2013 [1], les études montrent que le risque moyen de second cancer primitif (SCP) après un premier diagnostic de cancer chez l'adulte, apparaît légèrement plus élevé que le risque de cancer dans la population

\section{M.-F. Bacqué ( $\square)$}

SULISOM, université de Strasbourg,

12, rue Goethe, F-67000 Strasbourg, France

e-mail : mfbacque@club-internet.fr

\section{A. Polomeni $(\bowtie)$}

Service d'hématologie clinique et thérapie cellulaire, hôpital Saint-Antoine, Assistance publique-Hôpitaux de Paris, 184, rue du Faubourg-Saint-Antoine, F-75012 Paris, France e-mail : alice.polomeni@aphp.fr

\section{Derzelle ( $\square)$}

Institut Jean-Godinot, 1,

avenue du Général-Kœnig, F-51100 Reims, France

e-mail : martine.derzelle@reims.unicancer.fr

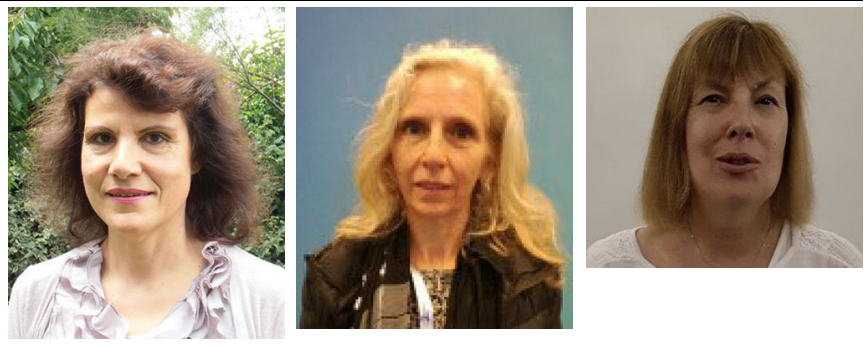

générale. Les leucémies induites par les traitements représenteraient $7 \%$ de l'ensemble des leucémies aiguës myéloblastiques (LAM) et surviendraient, en médiane à 70 ans, soit un âge similaire aux autres LAM. Les données de suivi après dix ans montrent que le risque de développer une leucémie induite est de 2,15 cas pour 1000 femmes traitées pour un cancer du sein et de 5,8 cas pour 1000 personnes traitées pour une maladie de Hodgkin. Des chiffres toutefois non négligeables...

Un SCP est une nouvelle tumeur primitive infiltrante diagnostiquée chez un individu déjà atteint par un cancer. Cette nouvelle tumeur n'est ni une récidive, ni une métastase, ni un cancer multifocal ou multicentrique [1].

Face à ces inconnues et à la difficulté à accepter non pas la rechute, non pas la poursuite des traitements à vie, mais l'apparition de ce qui est bien une autre maladie, oncologues et psychologues s'allient pour aborder dans ce numéro de Psycho-Oncologie, les écueils les plus importants :

- comment annoncer un second cancer à un patient ?

- comment prévoir la possibilité d'un second cancer et l'aborder dans la population générale ou seulement chez les malades ?

- comment développer le dépistage d'un second cancer ?

- comment prévenir concrètement le risque de second cancer chez les personnes guéries, surtout chez celles qui ont développé leur première maladie très jeunes?

\section{Réactions émotionnelles intenses}

Sandra Malak, hématologue à l'hôpital René-Huguenin, institut Curie de Saint-Cloud, aborde les aspérités de la relation médecin-malade lorsque survient une leucémie aiguë radioou chimio-induite après les traitements d'un cancer. Elle reconnaît l'insuffisance actuelle de la confirmation de liens de causalité entre traitements et apparition du second cancer. L'hypothèse principale à ce jour reste celle de l'émergence de cellules anormales liées à l'instabilité génétique due aux traitements anticancéreux. 
Les conséquences émotionnelles de cette situation sont lourdes. Les médecins ressentent souvent une certaine culpabilité. La proposition thérapeutique était-elle bien adaptée ? Dans le cas contraire, le principe du Primum non nocere est mis à mal. Si le même médecin a suivi le malade lors de son premier cancer et prolonge son accompagnement, l'alliance thérapeutique ne risque-t-elle pas d'être fragilisée ? Une nouvelle équipe n'est-elle pas plus indiquée pour limiter les implications conscientes et inconscientes négatives ? Et que dire de tout cela si, précisément, les risques de second cancer n'avaient pas été abordés ?

Les conséquences les plus fréquentes semblent toucher la confiance et l'énergie des patients, la frustration, l'angoisse et la culpabilité pour les médecins. Les psychologues Malika Aïnaoui-Bertoux et Carole Augé partagent avec le corps soignant les réactions des patients souvent tétanisés par le second cancer. Ils vivent un nouveau cauchemar et expriment des sentiments d'injustice, une extrême vulnérabilité, une perte totale d'espoir. "Invraisemblable, impensable! J'en arrive à penser que je serais mieux morte! » s'exclame une patiente à l'idée d'annoncer à sa famille sa deuxième maladie.

Autant les médecins sont sensibilisés à l'annonce d'un cancer, autant la succession, voire le cumul de ces situations, n'a pas été totalement investiguée, même si nous savons que la multiplication des deuils par exemple, est un facteur d'aggravation psychopathologique.

Des oncologues proposent une consultation conjointe entre médecins (du premier et du second cancer) afin de mieux aménager et supporter les réactions extrêmes des patients et de leurs proches. Cette situation semble en effet à haut risque de décompensation, aussi vaut-il mieux partager calmement la charge psychique et trouver des partenaires susceptibles de représenter, pour le patient et ses proches, un nouveau soutien psychologique face à l'épreuve.

S'entendre annoncer un deuxième cancer, souvent quelques années après le premier, constitue une nouvelle effraction qui réactive une quête de sens, selon les psychologues, au sujet du corps défaillant.

À l'inverse, il existe des réactions de toute puissance. Certains patients pensent bien maitriser la situation puisqu'ils ont déjà traversé le temps chaotique du premier cancer.

\section{Mais comment prévoir sans faire peur?}

Bénédicte Bruno, médecin hématologue en pédiatrie, évoque les contraintes du dépistage systématique (et à vie) d'un potentiel second cancer chez de jeunes enfants traités pour leucémie. Alors que cinq années après la greffe, le médecin peut annoncer sa guérison à l'enfant et à ses parents, il doit aussi leur faire accepter la recherche annuelle de cancers secondaires aux traitements. L'incidence du cancer de la thyroïde vingt ans après une greffe de moelle avec irradiation est de $14 \%$, tandis que l'incidence du cancer de la peau (hors mélanome) après cette greffe est encore de $8 \%$. Le dépistage, simple, consiste en une échographie thyroïdienne annuelle et une consultation annuelle en dermatologie. Par ailleurs ces deux cancers, s'ils sont dépistés à temps, donnent lieu à des traitements simples, en comparaison de ceux de la leucémie et avec une bonne efficacité. Les médecins hématologues se trouvent donc dans une situation délicate : comment parler (déjà ?) du risque de cancer secondaire alors que l'enfant et sa famille affrontent encore la leucémie initiale ?

La période la plus difficile sera peut-être l'adolescence de ces jeunes qui souhaitent comme tous les autres, ressentir la liberté, l'autonomie et le choix de leur vie. La visite annuelle leur rappelle le contraire : le risque mortel, la dépendance aux examens médicaux, une vie limitée par une relative incertitude. Non, décidément, le médecin n'augure pas de bonnes nouvelles lorsqu'il représente ainsi le principe de réalité.

Mariane Lecointe et Nathalie Dumet proposent un dispositif d'accompagnement original pour permettre à une patiente de supporter son second cancer. Par la médiation de la socioesthétique incluse dans les soins de support, une psychologue clinicienne et une socioesthéticienne permettent à un groupe de patientes de s'approprier leur nouvel état et, par là leur nouveau « psychosoma », terme proposé par Joyce McDougall pour désigner les liens inconscients entre le corps et l'esprit. La médiation en six séances apporte un retour des identifications aux femmes du groupe, une possibilité de sublimer ses désirs sexuels par le biais du maquillage, mais surtout l'expression mentalisée ${ }^{1}$ de la honte transgénérationnelle liée à son histoire migratoire et potentiellement les fantasmes d'être rattrapée par la génétique familiale alors qu'elle fuyait ses racines. Le deuxième cancer a en effet cette force; il se fait l'interprète tout-puissant de ce qui avait pu être refoulé. À l'occasion de sa survenue si brutale, toute rationalité peut disparaître et ne laisser que la pensée primitive du nourrisson.

Face à cette déflagration du nouveau cancer, PsychoOncologie, en traitant ce sujet rare, a choisi de vous permettre de réfléchir à ces cas qui entraînent tant de sentiments et d'émotions complexes. La durée de survie de nos patients est un bienfait, elle met seulement en évidence quelques nouveaux risques, le second cancer est l'un d'eux.

\section{Références}

1. INCa (2013) Identifier et prévenir les risques de second cancer primitif chez l'adulte, collection État des lieux et des connaissances, ouvrage collectif, Boulogne-Billancourt

\footnotetext{
${ }^{1}$ La mentalisation est la capacité de prendre conscience de ses propres états psychiques et de ceux d'autrui. Il s'agit de "penser ce que l'on ressent et de sentir ce que l'on pense ", d'après Fonagy P, Bateman A. Handbook of Mentalizing in Mental Health Practice (2012). Ed by Peter Fonagy and Anthony Bateman. American Psychiatric Publishing. New York London.
} 